\title{
ON THE CHARACTERISTIC FUNCTION OF A SUM OF M-DEPENDENT RANDOM VARIABLES
}

\author{
WANSOO T. RHEE \\ Faculty of Management Sciences \\ The Ohio State Uni versity \\ Columbus, Ohio 43210
}

(Received October 8, 1985)

ABSTRACT. Let $\mathrm{S}=\mathrm{f}_{1}+\mathrm{f}_{2}+\ldots+\mathrm{f}_{\mathrm{n}}$ be a sum of 1 -dependent random variables of zero mean. Let $\sigma^{2}=E S^{2}, L=\sigma^{-3} \sum_{1 \leq i \leq n} E\left|f_{i}\right|^{3}$. There is a universal constant a such that for $a|t| L<1$, we have

$$
\left|E \exp \left(i t S \sigma^{-1}\right)\right| \leqq(1+a|t|) \sup \left\{(a|t| L)^{-1 / 4 \ln L}, \exp \left(-t^{2} / 80\right)\right\} \text {. }
$$

This bound is a very useful tool in proving Berry-Esseen theorems.

KEY WORDS AND PHRASES. Characteristic Function, m-dependent random variable, Berry-Esseen bound.

1980 AMS SUBJECT CLASSIFICATION CODE. P60F05.

1. INTRODUCTION.

Consider a sequence of independent random variables $f_{1}, f_{2}, \ldots, f_{n}$ of zero means having third moments. Let $S=f_{1}+\ldots+f_{n}$ and $\sigma^{2}=E S^{2}$.

If $t \leqq \sigma E\left(f_{i}^{2}\right) / E\left|f_{i}\right|^{3}$ for each $i \leqq n$, one has

$$
\begin{aligned}
\left|E\left[\exp \left(i t S \sigma^{-1}\right)\right]\right| & \leqq \prod_{i \leq n}\left|E\left[\exp \left(i t f_{i} \sigma^{-1}\right)\right]\right| \leq \prod_{i \leq n} \exp \left(-t^{2} / 3 \operatorname{Ef}_{i}^{2} \sigma^{-2}\right) \\
& \leqq \exp \left(-t^{2} / 3\right) .
\end{aligned}
$$

This trivial estimate plays a fundamental role in the proof of Berry-Esseen rates of convergence in the central limit theorem. The purpose of this work is to $f$ ind an estimate of $\left|E\left[\exp \left(i t S \sigma^{-1}\right)\right]\right|$ for the sequence of $m$-dependent random variables.

We say that a sequence $\left(f_{i}\right)_{i=1}^{n}$ of random variables is m-dependent if for each $1 \leqq p \leqq n-m-1$, the sequences $\left(f_{i}\right)_{i \leqq p}$ and $\left(f_{i}\right)_{i>p+m}$ are independent of each other.

In a recent very interesting paper by $V$. V. Shergin[1], the author gives the best rate of convergence in the central limit theorem for $m$-dependent random 
variables. We will estimate the bound of $\left|E\left[\exp \left(i t S \sigma^{-1}\right)\right]\right|$ by Shergin's methods. This result extracts the most important ideas of Shergin's work. Also we want to point out that this estimate turns out to be an essential tool in the proof of BerryEsseen type bounds in other limit theorems for $m$-dependent random variables. In a subsequent work, we shall establish such a convergence rate for U-statistics[2] and an Edgeworth expansion for a sum of $\mathrm{m}$-dependent random variables[3].

2. CONSTRUCTION.

We follow the lines of Shergin's ingenious construction to decompose $S$ in an amenable way. We do not however assume the reader to be familiar with Shergin's paper. The exposition is self cuntalned, and some long details of $h ; p r$, of ar: eliminated by our approach.

We assume now on $m=1$. We denote $a_{0}, a_{1}, \ldots, a_{\ell}$ universal constants. No attempt is made at finding optimal values for these universal constants, since the numerical values involved here are too large to be of any interest.

Set $U=\sum_{i \leq n} E\left|f_{i}\right|^{3}, L=U \sigma^{-3}$ and $R=-$ In $L$. In the sequel, we assume $R \geq 10$. It follows that for $i \leq n$, we have

$$
E f_{i}^{2} \leq\left(E\left|f_{i}\right|^{3}\right)^{2 / 3} \leq \sigma^{2} L^{2 / 3} \leq \sigma^{2} / 50 R
$$

By induction we define indices $s(i)$ as follows. Set $s(1)=1$, and

$$
s(i+1)=1+\min \left\{s: s>s(i), E\left(f_{s(i)}+\ldots+f_{s}\right)^{2} \geq \sigma^{2} / R\right\} .
$$

The construction stops at an index $h$ such that either $s(h)=n$ or $E\left(f_{s(h)}+\ldots+f_{s}\right)^{2}<\sigma^{2} / R$ for $s(h)<s \leqq n$.

LEMMA 1. ([1]) We have $10 R / 11 \leq h \leq 2 R$ and $s(i+1)-s(i) \geq 15 R$ for $1 \leq i \leq h-1$.

PROOF. From the 1 -dependence of the $f_{i}$ it follows that

$$
\begin{aligned}
\sigma^{2}=\sum_{i=1}^{n-1} E\left(f_{s}(i)\right. & \left.+\ldots+f_{s(i+1)-1}\right)^{2}+E\left(f_{s}(h)+\ldots+f_{n}\right)^{2} \\
& +2 \sum_{i=1}^{h-1} E\left\{f_{s(i+1)-1} f_{s(i+1)}\right\} .
\end{aligned}
$$

It follows from Schwartz's inequality and (2.1) that

$$
\sigma^{2} \geq(n-1) \sigma^{2} / R-2(n-1) \sup E\left|f_{i}\right|^{2} \geq 24 \sigma^{2}(n-1) / 25 R
$$

so that $h \leqq 25 R / 24+1 \leqq 2 R$. Mor eover

$$
\begin{gathered}
\sigma^{2}=\sum_{i=1}^{n-1} E\left(f_{s(i)}+\ldots+f_{s(i+1)-2}\right)^{2}+E\left(f_{s(n)}+\ldots+f_{n}\right)^{2} \\
+\sum_{i=1}^{n-1} E f_{s(i+1)-1}^{2}+2 \sum_{i=1}^{n-1} E\left(f_{s}(i+1)-2^{f} s(i+1)-1\right)+2 \sum_{i=1}^{n-1} E\left(f_{s(i+1)-1} f_{s(i+1)}\right) .
\end{gathered}
$$

So, 


$$
\sigma^{2} \leqq h \sigma^{2} / R+5 h \text { sup } E f_{i}^{2} \leq 11 h \sigma^{2} / 10 R
$$

and hence $h \geq 10 R / 11$. On the other hand, for $i \leq h-1$,

$$
\begin{aligned}
\sigma^{2} / R & \leqq E\left(f_{s(i)}+\ldots+f_{s(i+1)-1}\right)^{2}=\sum_{j=s(i)}^{s(i+1)-1} E f_{j}^{2}+\sum_{j=s(i)}^{s(i+1)-2} 2 E\left(f_{j} f_{j+1}\right) \\
& \leq 3(s(1+1)-s(i)) \operatorname{Max} E f_{i}^{2} \leq 3(s(i+1)-s(i)) \sigma^{2} / 50 R
\end{aligned}
$$

whlch proves the lemma.

Q.E.D.

$$
\text { For } i \leqq h-1 \text {, let } \tau_{i}=\sum_{j=s(i)}^{s(i+1)-1} E\left|f_{j}\right|^{3} \text {. We have } \sum_{i=1}^{n-1} \tau_{i} \leq U \text {. Hence if } p \text { is the number }
$$

of indices $i \leq h-1$ such that $\tau_{i} \geq 10 U(h-1)^{-1}$, we have $p \leq(h-1) / 10$. It follows that there are at least $9(\mathrm{~h}-1) / 10$ indices $i$ for which $\tau_{1} \leq 10 \mathrm{U}(\mathrm{h}-1)^{-1}$.

Let $H=[9(h-1) / 20-1]$. If $R \geq 10$, we have $H \geq R / 10$. This follows from the fact that $h \geqq 10 R / 11$ and straightforward computations. We can moreover select indices $i_{1}, \ldots, i_{H}$ such that for $1 \leqq \ell \leqq h$,

$$
\tau_{i_{\ell}} \leq 10 U(h-1)^{-1} \leq 20 U / R ; i_{\ell+1}-i_{\ell} \geq 2,2 \leq i_{\ell} \leq n-2 .
$$

For $1 \leqq \ell \leqq H$, we set $s\left(i_{\ell}\right)=a_{\ell}, s\left(i_{\ell}+1\right)=a_{\ell}^{\prime}$. We have $a_{\ell}-a_{\ell}^{\prime} \geq 15 R \geq 15 H$. Let

$$
\overline{\mathrm{f}}_{\ell}=\left(a_{\ell}^{\prime}-a_{\ell}\right)^{-1} \sum_{a_{\ell} \leq j<a_{\ell}^{\prime}} E\left|f_{j}\right|
$$

Since there are at least $15 \mathrm{H} / 2 \geqq 7 \mathrm{H}$ indices $a_{\ell} \leq j<a_{\ell}^{\prime}$ for which $E\left|f_{j}\right| \leq 2 \bar{f}_{\ell}$, one can pick indices $\mathrm{p}(\ell,-H), \ldots, \mathrm{p}(\ell, 0), \ldots, \mathrm{p}(\ell, H)$ of $\left[a_{\ell}, a_{\ell}^{\prime}[\right.$ with this property such that no two of them are consecutive.

LEMMA 2. For each $-\mathrm{H} \leq \mathrm{i} \leq \mathrm{H}$, we have $E\left|f_{p(\ell, i)}\right| \leq 40 \mathrm{~L}$.

PROOF. By Holder's inequality, we have,

$$
\begin{aligned}
& \sum_{a_{\ell} \leqq j<a_{\ell}^{\prime}} F\left|f_{j}\right| \leq \sum_{a_{\ell} \leq j<a_{\ell}^{\prime}}\left(E\left|f_{j}\right|^{3}\right)^{1 / 3} \leq\left(a_{\ell}^{\prime}-a_{\ell}\right)^{2 / 3} \tau_{i}^{1 / 3}, \\
& \sum_{\ell \leq j<a_{\ell}^{\prime}} E\left|f_{j}\right|^{3} \leq \sum_{a_{\ell} \leq j<a_{\ell}^{\prime}}\left(E\left|f_{j}\right|^{3}\right)^{2 / 3} \leq\left(a_{\ell}^{\prime}-a_{\ell}\right)^{1 / 3} \tau_{i}^{2 / 3} .
\end{aligned}
$$

As already shown, $E\left(\underset{a_{\ell} \leq j<a_{\ell}^{\prime}}{\sum} f_{j}\right)^{2} \leqq 5 a_{\ell} \leq j<a_{\ell}^{\prime} \sum_{j}^{2}$, so we get by combining the above inequalities, and since $\sigma^{2} \leqq R E\left(\sum_{a_{\ell} \leq j<a_{\ell}^{\prime}} f_{j}\right)^{2}, \sigma^{2} \bar{f}_{\ell} \leq 2 R \tau_{i_{\ell}} \leq 40 U$.

Q.E.D.

Set 


$$
\begin{gathered}
z_{\ell, 0}=f_{p(l, 0)}, z_{2 q}=f_{p(l,-q)}+f_{p(l, q)} \text {, for } 0: q \leq H, \\
z_{2 q+1}=_{p(l,-q-1)<i<p(l,-q)} f_{i}+\sum_{p(l, q)<i<p(\ell, q+1)} f_{i}, \text { for } 0 \leq q \leq H .
\end{gathered}
$$

For $1 \leq s \leq H$, let $b_{s}=\left(e_{1}, \ldots, e_{s}\right)$ be a collection of integers $0 \leq e_{s} \leq 2 H+1$. We set

$$
W\left(b_{s}\right)=\sigma^{-1}\left(s-\sum_{\ell=1} \sum_{j \leq e_{s}} z_{\ell, j}\right) .
$$

For $s<H, \ell \mathrm{H}_{1}, b_{s}=\left(e_{1}, \ldots, e_{s}\right)$, we set $W\left(b_{s}, l\right)=W\left(\left(e_{1}, \ldots, e_{s}\right), l\right)$. Let

$$
\phi_{s}(t)=\max \left|E\left[\exp \left(i t-W\left(b_{s}\right)\right)\right]\right|
$$

and if $s<H$, let

$$
\lambda_{s}(t)=\max \left|E\left[\exp \left(i t W\left(b_{s}\right)\right)-\exp \left(i t W\left(b_{s}, 0\right)\right)\right]\right| .
$$

Here the maximum is taken over all possible choices of $b_{s}$.

3. ESTIMATES.

LEMMA 3. There is a universal constant $a_{1}$ such that for $a_{1}|t| L<1$ and $1 \leq a<H$ we have

$$
\lambda_{s}(t) \leq\left(a_{1}|t| L\right)^{H+1}+a_{1}|t| L \phi_{S+1}(t) .
$$

PROOF. Let us $\mathrm{fix} b_{S}$, and for $0 \leq k \leq 2 \mathrm{H}+1$, let $\gamma_{k}=\exp \left(i t z Z_{\left.s, k^{\sigma^{-1}}\right)-1}\right.$.

one sees by induction that

$$
\begin{aligned}
\exp \left(i \operatorname{tw}\left(b_{s}\right)\right)-\exp \left(i \operatorname{tw}\left(b_{s}, 0\right)\right)=\sum_{\ell=1}^{2 H+1} \exp \left(i \operatorname{tW}\left(b_{s}, l\right)\right) \prod_{k=0}^{\ell-1} \gamma_{k} \\
+\exp \left(i \operatorname{tw}\left(b_{\ell}, 2 H+1\right) \prod_{k=0}^{2 H+1} \gamma_{k} .\right.
\end{aligned}
$$

By a well known estimate and lemma 2, we have

$$
E\left|\gamma_{2 r}\right| \leq|t| \sigma^{-1} E\left|z_{r}\right| \leq 80|t| L \text { for } 0 \leq 2 r \unlhd H-1 \text {. }
$$

So, since $\left|\gamma_{k}\right| \leq 2$, if we set $\bar{\ell}=[\ell / 2]$, we have

$$
E\left|\prod_{k=0}^{l-1} \gamma_{k}\right| \leq 2^{l} E \prod_{i \leq \bar{l}}\left|\gamma_{21}\right| \leq(160|t| L)^{\bar{l}+1} .
$$

Thus the lemma follows (with $a_{1}=320$ ) by taking expectation in (3.1), and since

$$
\begin{aligned}
& \sum_{l=0}^{\infty}(160|t| L)^{\bar{\ell}+1} \leq 2 \text { for } a_{1}|t| L<1 . \\
& \text { LEMMA 4. } \phi_{H}(t) \leq\left(\exp \left(-t^{2} / 4 R\right)+a_{4}|t| L\right)^{H} . \\
& \text { PROOF. We fix } b_{H}=\left(e_{1}, \ldots, e_{H}\right) \text {. For } 1 \leq l \leq H \text {, we set } \\
& \quad r_{\ell}=p(\ell,-q)-1, r_{\ell}^{\prime}=p(l, q)+1 \text { if } e_{\ell} \text { is of the form 2q }
\end{aligned}
$$

and 


$$
r_{\ell}=p(l,-q-1), r_{l}^{\prime}=p(x, q-1) \text { if } e_{\ell} \text { is of the form } 2 q+1 \text {. }
$$

Let $r_{0}^{\prime}=1$ and $r_{H+1}=n$. For $0 \leq \ell \leq H$, let

$$
T_{\ell}=\sum_{r_{\ell}^{\prime} \leq i \leq r_{\ell+1}} f_{i} .
$$

We have $W\left(b_{H}\right)=\sigma^{-1}\left(T_{0}+\ldots+T_{H}\right)$, and the $T_{\ell}$ are independent. Moreover it follows from (2.2) that each $T_{\ell}$ is the sum of the $f_{i}$ over an interval which contains an interval of the type $\left[s\left(j_{\ell}\right), s\left(j_{\ell}+1\right)[\right.$. It follows that

$$
\begin{aligned}
& \sigma_{\ell}^{2}=E\left|T_{\ell}\right|^{2} \geqq E\left(\sum_{s\left(j_{\ell}\right) \leq i<s\left(j_{\ell}+1\right)} f_{i}\right)^{2}-2 E\left(f_{s}\left(j_{\ell}\right) f_{s\left(j_{\ell}-1\right)}\right)-2 E\left(f_{s}\left(j_{\ell}+1\right) f_{s}\left(j_{\ell}+2\right)\right) \\
& \geqq \sigma^{2} / 2 R \text {. }
\end{aligned}
$$

Let $\omega_{\ell}=\sum_{r_{\ell}^{\prime} \leq i \leqq r_{\ell+1}} E\left(f_{i}\right)^{3}$. It follows from the theorem of $R$. V. Erickson [4] that for each $z, \mid E\left(\exp\right.$ izT $\left._{\ell} \sigma_{\ell}^{-1}\right)-\exp \left(-z^{2} / 2\right)\left|\leqq a_{3}\right| z \mid \omega_{\ell} \sigma_{\ell}^{-3}$.

By taking $z=t_{\sigma_{\ell}} \sigma^{-1}$ and using $\sigma_{\ell}^{2} \geq \sigma^{2} / 2 R$, one gets

$$
\left|E\left(\exp i t T_{\ell} \sigma^{-1}\right)\right| \leq \exp \left(-t^{2} / 4 R\right)+2 a_{3} \sigma^{-3} R \omega_{\ell} .
$$

Thus, we get

$$
\mid E\left(\exp i t W\left(b_{H}\right) \mid \leq \prod_{\ell=q}^{H}\left(\exp \left(-t^{2} / 4 R\right)+2 a_{3} \sigma^{-3} R \omega_{\ell}\right) .\right.
$$

The concavity of the function $\ln (1+x)$, and the fact that $\sum_{\ell=0}^{H} \sigma^{-3} R \omega_{\ell} \leq \mathrm{RL} \leq 10 \mathrm{HL}$ prove the result.

Q.E.D.

4. RESULTS.

PROOSITION 5. If $a_{5}|t| L \leqq 1$ (and $L \leqq e^{-10}$ ), we have

$$
\left|E\left(\exp i t S \sigma^{-1}\right)\right| \leq\left(1+a_{5}|t|\right)\left(\exp \left(-t^{2} / 4 R\right)+a_{5}|t| L\right)^{H} \text {. }
$$

PROOF. Since $\phi_{S} \leqq \phi_{S+1}+\lambda_{S+1}$, it follows easily from lemma 3 and by induction that

$$
\begin{aligned}
& \sum_{l=1}^{q} \lambda_{l}(t) \leqq q\left(1+a_{1}|t| L\right)^{q}\left(\left(a_{1}|t| L\right)^{H+1}+a_{1}|t| \phi_{s+1}(t)\right) . \\
& \left.\mid E(c x) i \operatorname{tso} \sigma^{-1}\right) \mid \leqq \phi_{H^{+}} \sum_{s=1}^{H} \lambda s \\
& \leqq \phi_{H}(t)\left(1+H a_{1}|t| L\left(1+a_{1}|t| L\right)^{H}\right)+H\left(1+a_{1}|t| L\right)^{H}\left(a_{1}|t| L\right)^{H}\left(a_{1}|t| L\right)^{H+1} .
\end{aligned}
$$$$
\text { So, }
$$ 
If $a_{1}|t| L \leq e^{1 / 2}-1$, we have, since $H \leq R=\ln L^{-1}$,

$$
H\left(1+a_{1}|t| L\right){ }^{H} \leqq 2\left(\ln L^{-1}\right) L^{-1 / 2} \leq a_{\sigma} L^{-1} \text {. }
$$

So proposition 5 follows from lemma 4 with $a_{5}=\sup \left(10 a_{1}, 2 a_{3}, a_{\sigma}\right)$.

It is well worthwhile to reformulate the above result to show more precisely the behaviour of the bound.

THEOREM 6. There exists universal constants $a_{7}$ and $a_{8}$ such that for $q \varepsilon \mathbf{I}$ and $|t|, a_{7} \leqq|t|$ and $a_{8}|t| L \leqq 1$, and

$$
\left|E\left(\exp i t \sigma^{-1}\right)\right| \leqq\left(1+a_{5}|t|\right) \sup \left(\exp \left(-t^{2} / 80\right),\left(a_{8}|t| L\right)^{\ln L}\right) .
$$

PROOF. Let $a_{8}=3 a_{5}$. By taking $a_{7}$ large enough, the existence of one $|t|$ satisfying the hypothesis implies $L \geq e^{10}$, so we can assume that (4.1) holds. We can also assume that $a_{7} \geq 80 a_{5}$. If $|t| \leqq 2 \sqrt{R}$, we have $\exp \left(-t^{2} / 4 R\right) \geq 1 / e$. Thus, since $\mathrm{H} \geqslant \mathrm{R} / 10$,

$$
\begin{gathered}
\left(\exp \left(-t^{2} / 4 R\right)+a_{5}|t| L\right)^{H} \leqq \exp \left(-t^{2} / 40\right)(1+e a|t| L)^{H} \\
\leqq \exp \left(-t^{2} / 40+e a_{5}|t| L H\right) .
\end{gathered}
$$

Since $L H \leqq L R \leqq 1 / e$ and $|t| \geqq 80 a_{5}$, we have $-t^{2} / 40+e a_{5}|t| L H \leq-t^{2} / 80$, which proves (4.2) in that case. If $t \geq 2 \sqrt{R}$, then $\exp \left(-t^{2} / 8 R\right) \geq \sqrt{e}\left(\exp \left(-t^{2} / 4 R\right)\right)$, and it is easy to check that

$$
\exp \left(-t^{2} / 4 R\right)+a_{5}|t| L \leq \operatorname{Max}\left\{\exp \left(-t^{2} / 8 R\right), 3 a_{5}|t| L\right\}
$$

and theorem 6 follows.

REMARK. (1) In case of a $m$-dependent ( $m>1)$ sequence of random variables, an estimate of $\left|E\left(\exp i t S \sigma^{-1}\right)\right|$ can be obtained by considering $S$ as the sum of 1dependent blocks of $\mathrm{f}_{i}$.

(2) The constant $1 / 4$ in the exponent of (4.2) plays no particular role. It is clear from the method that it can be replaced by any number; but the values of $a_{5}$ and $a_{8}$ depend on this exponent. However for the applications we have in mind, any positive number will be sufficient.

To support our claim that theorem 6 is useful tool, we deduce Shergin's theorem in a simpler way. Let $\Phi$ be the distribution function with the normal law.

SHERGIN'S THEOREM.

$$
\sup _{t}|P(S<t)-\Phi(t)| \leq a L .
$$

PROOF. It is possible either to use the construction of sections 2 and 3 or to do again a similar but much simpler construction. In order not to repeat arguments 
already used, we choose the first approach. Let $q=[H / 2]$ and $p=p(q, 0)$. Let $S_{1}=$ $\underset{0 \leq i<p}{f_{i}}$ and $s_{2}=\sum_{p<i \leq n} f_{i}$. For $s\left(i_{\ell}\right) \leq s \leq p\left(\right.$ resp. $\left.p<s \leq s\left(i_{\ell+1}\right)\right)$, it is easily seen that $E\left(\sum_{1 \leq i \leq s} f_{i}\right)^{2} \geq \sigma^{2} / 10$ (resp. $\left.E\left(\sum_{1 \leq i \leq n} f_{i}\right)^{2} \geq \sigma^{2} / 16\right)$. The method of 1 emma 3 and the result of theorem 6 gives for $4 a_{8}|t| L \leq 1$ :

$$
\begin{gathered}
\mid E\left(\exp i t S \sigma^{-1}\right)-E\left(\exp i t\left(S_{1}+S_{2}\right) \sigma^{-1} \mid\right. \\
\leqq\left(a_{1}|t| L\right)^{H+1}+a_{1}|t| L\left(1+4 a_{5}|t|\right) M a x\left\{\exp \left(-t^{2} / 320\right),\left(4 a_{8}|t| L\right)^{-\frac{1}{4} \ln L / 64}\right\} .
\end{gathered}
$$

Moreover, if $\sigma_{1}^{2}=\mathrm{ES}_{1}^{2}$ and $\sigma_{2}^{2}=\mathrm{ES}_{2}^{2}$, we get for $\varepsilon=1,2$, from Erickson's theorem:

$$
\left|E\left(\exp i t s_{\varepsilon} \sigma^{-1}\right)-\exp \left(-t^{2} \sigma_{\varepsilon}^{2} / 2 \sigma^{2}\right)\right| \leq 16 a_{3}|t| L .
$$

So it follows, using again theorem 6 , that

$$
\begin{gathered}
\mid E\left(\exp \text { it }\left(S_{1}+S_{2}\right) \sigma^{-1}\right)-\exp \left(-t^{2}\left(\sigma_{1}^{2}+\sigma_{2}^{2}\right) / 2 \sigma^{2}\right) \mid \\
\leqq 36 a_{3}|t| L\left(1+4 a_{5}|t|\right) M a x\left\{\exp \left(-t^{2} / 320\right),\left(4 a_{8}|t| L\right)^{-\frac{1}{4} \ln L / 64}\right) .
\end{gathered}
$$

Now, if we set $T^{-1}=4 e^{8} a_{8} L$, a straightforward computation gives

$$
J(T)=\int_{-T}^{T} t^{-1}\left|E(\exp i t S)-\exp \left(-t^{2}\left(\sigma_{1}^{2}+\sigma_{2}^{2}\right) / 2 \sigma^{2}\right)\right| \leq a_{10} L .
$$

The familiar Esseen inequality gives

$$
\sup _{x}\left(P\left(S \sigma^{-1}<x\right)-\Phi^{\prime}(x)\right) \leq a\left(J(T)+T^{-1}\right) \leq a_{11} L
$$

where $\Phi^{\prime}(x)$ is the normal distribution function with variance $k^{2}=\sigma^{-2}\left(\sigma_{1}^{2}+\sigma_{2}^{2}\right)=\sigma^{-2} E\left(S-f_{p}\right)^{2}$. We have

$$
\sup _{x}\left(\Phi^{\prime}(x)-\Phi(x)\right) \leq 1-k^{2} \leq \sigma^{2}\left(E\left(f_{p}^{2}\right)+2 E\left(f_{p-1} f_{p}\right)+2 E\left(f_{p} f_{p+1}\right)\right) .
$$

We can also assume that at the time we picked the indices $p(q, j)$ we have made the extra effort to choose $p=p(q, 0)$ such that for $-=1,0,1$, we have $E\left(f_{p+\varepsilon}^{2}\right) \leq 10\left(a_{q}^{\prime}-a_{q}\right) \sum_{\ell \leq j \leq a_{\ell}^{\prime}} E\left|f_{j}\right|^{2}$. It then follows by an estimate similar to lemma 2 that the right hand side of the parenthesis is also bounded by $a_{12} L$, and concludes the proof.

REFERENCES

1. SHERGIN, V.V. On the convergence rate in the central limit theorem for $m$ dependent random variables, Theor. Prob. appl. XXIV (1979), 782-796. 
2. RHEE, W. On Asymptotic normality for m-dependent U-Statistics, to appear.

3. RHEE, W. An Edgeworth expansion for a sum of $m$-dependent random variables, to appear in International Journal of Math. and Mathematical Sci.

4. ERICKSON, R.V. $L_{1}$ bounds for asymptotic normality of m-dependent sums using Stein's technique, Ann. Prob. 2, 3 (1974), 522-529.

5. FELLER, W. An Introduction to Probability theory and its applications, John Wiley \& Sons, 1970. 


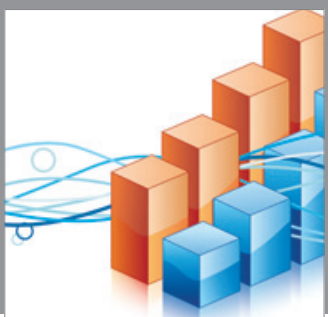

Advances in

Operations Research

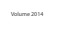

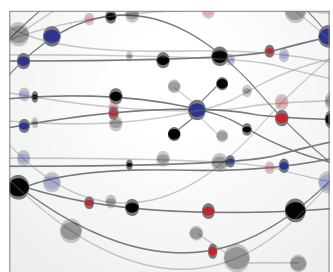

\section{The Scientific} World Journal
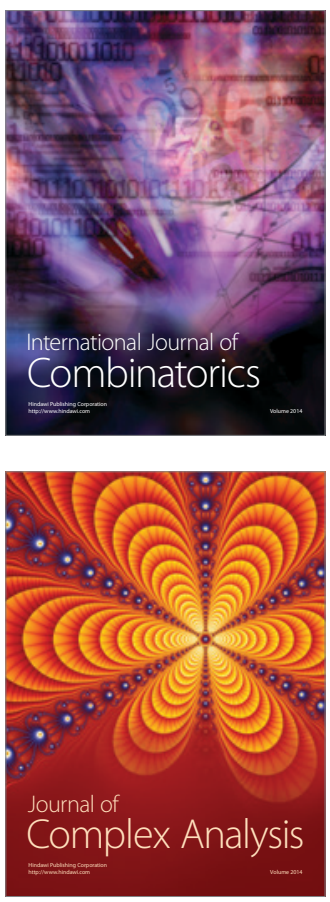

International Journal of

Mathematics and

Mathematical

Sciences
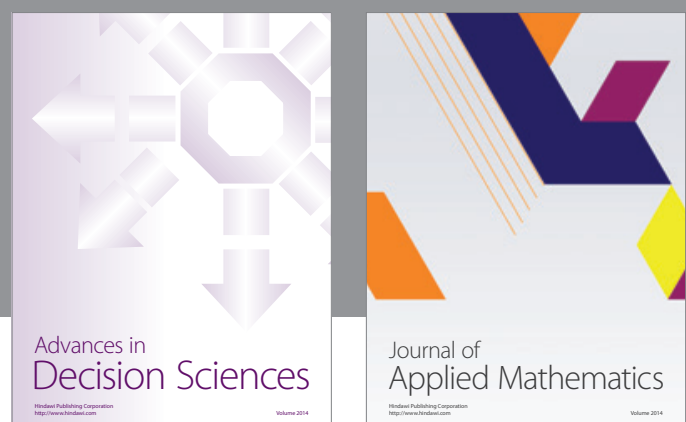

Journal of

Applied Mathematics
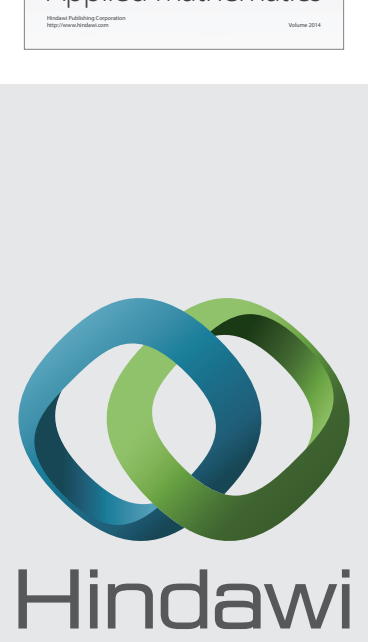

Submit your manuscripts at http://www.hindawi.com
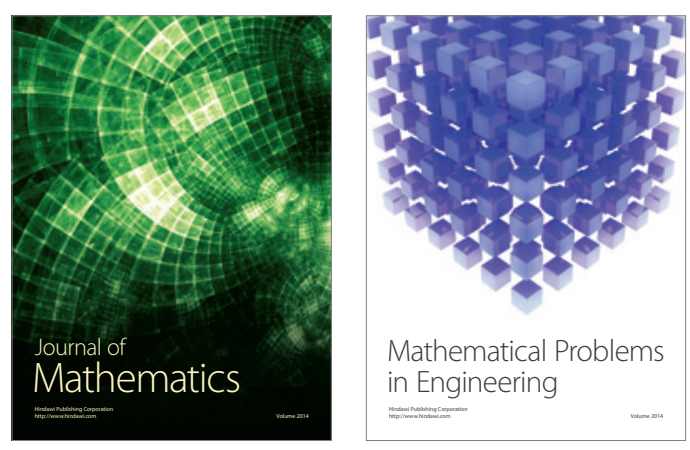

Mathematical Problems in Engineering
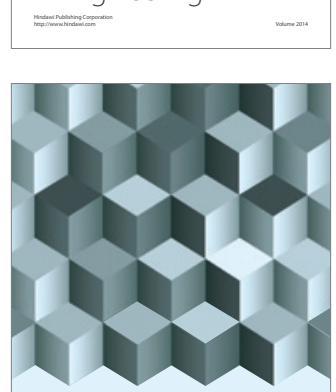

Journal of

Function Spaces
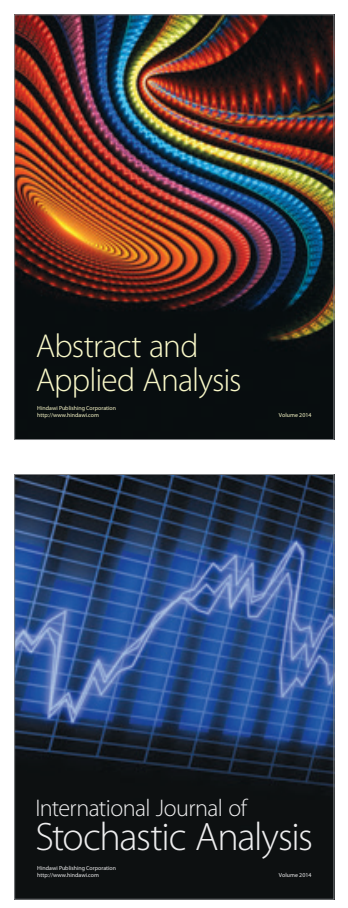

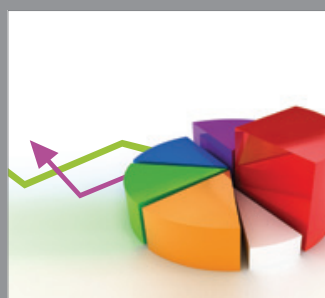

ournal of

Probability and Statistics

Promensencen
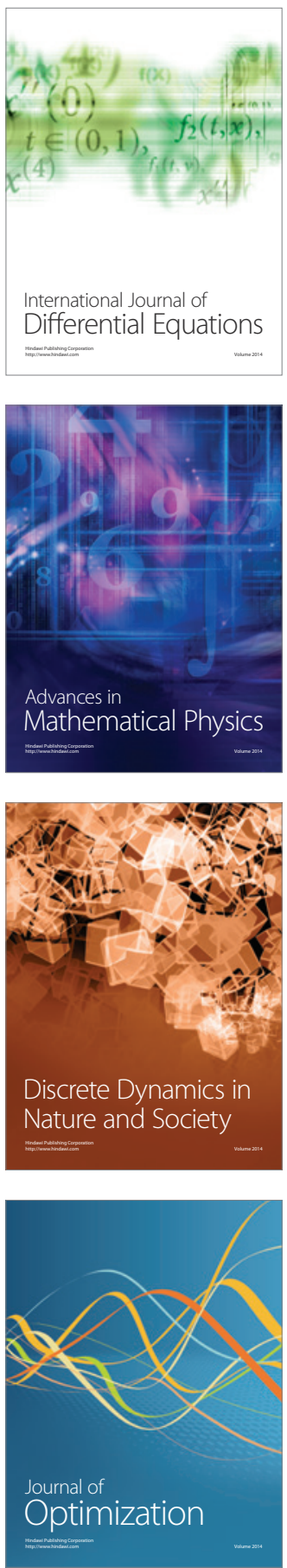